\section{Endobronchialventil reduziert Überblähung bei Lungenemphysem}

Kemp SV et al. A Multicenter Randomized Controlled Trial of Zephyr Endobronchial Valve Treatment in Heterogeneous Emphysema (TRANSFORM). Am J Respir Crit Care Med 2017; 196: $1535-1543$

An einer chronisch obstruktiven Lungenkrankheit (COPD) leiden weltweit Schätzungen zufolge etwa 300 Millionen Menschen. Liegt ein Lungenemphysem vor, gibt es die Option der chirurgischen Lungenresektion, eine Alternative stellen bronchoskopisch platzierte Endobronchialventile dar. Diese erlauben Sekretfluss und Luftstrom nach außen, aber blocken den Lufteinstrom in überblähte Lungenabschnitte. Den Ventiltypus Zephyr verglichen die Autoren mit der Standardtherapie beim Lungenemphysem.

Kemp und Kollegen randomisierten $>40$ jährige Ex-Raucher mit einer fortgeschrittenen COPD und heterogenem Emphysem für diese multizentrische, prospektive Studie. Eingeschlossen wurden Patienten mit einem $\mathrm{FEV}_{1}$ nach Bronchodilatation von $15-45 \%$ des Sollwerts trotz optimaler medikamentöser Therapie, deutlicher Erhöhung von Lungenkapazität und Residualvolumen sowie eingeschränkter Belastbarkeit im 6-Minuten-Gehtest (150-450 m Gehstrecke). Anhand eines speziellen Verfahrens (Chartis) ließ sich überprüfen, ob zwischen dem für das Endobronchialventil (EBV) infrage kommenden Lungenabschnitt und benachbarten Anteilen eine kollaterale Ventilation bestand. Nur dann, wenn dies nicht der Fall ist, ist ein EBV sinnvoll zu platzieren. Von 273 Probanden erfüllten 97 die beschriebenen Einschlusskriterien und wiesen keine Kollateralbelüftung auf. In einem Verhältnis von 2:1 erhielten schließlich 15 Patienten im Median 4 Ventile überwiegend in der rechten Lunge, die 32 anderen wurden weiterhin nach Standardempfehlungen behandelt.
Als primären Endpunkt definierten die Autoren eine Zunahme des FEV 13 Monate nach dem Eingriff um mindestens 12\% gegenüber dem Ausgangswert. Dieses Kriterium erfüllten 55,4\% der Patienten nach Intervention gegenüber 6,5\% der Kontrollen $(p<0,001)$. Sehr ähnliche Daten zeigten sich nach 6 Monaten. Bei 89,9\% der mit Ventil versorgten Patienten ließ sich eine Volumenreduktion in dem entsprechenden Lobus von mindestens $350 \mathrm{ml}$ erreichen. Sekundär bewerteten die Autoren zudem die Veränderungen des $\mathrm{FEV}_{1}$, der Strecke im 6-Minuten-Gehtest und der Ergebnisse in Scores zu Dyspnoe, Obstruktion und körperlicher Fitness im Verlauf der Studie. Alle diese Daten fielen für die Gruppe nach Intervention im Vergleich deutlich günstiger aus. Zu unerwünschten Wirkungen kam es bei $47,7 \%$ nach Platzierung der Ventile versus $9,4 \%$ in der Kontrollgruppe $(p<0,001)$. Neben Dyspnoe, Exazerbation oder Pneumonie kam es mit 29,2\% am häufigsten zu einem Pneumothorax bei den bronchoskopisch therapierten Patienten, der bei rund drei Viertel der Betroffenen per Intervention versorgt werden musste, in einem Fall chirurgisch. Ein Patient mit Pneumothorax starb an den Folgen eines Herzstillstands. Aus der Kontrollgruppe entschieden sich 94\% nach der 6-monatigen Beobachtung für die Versorgung mit einem Zephyr-Ventil.

FAZIT

Mit der multizentrischen Studie ließen sich erste positive Ergebnisse aus Untersuchungen an einzelnen Kliniken für den Einsatz von Zephyr-Endobronchialventilen bei heterogenem Lungenemphysem bestätigen. Die Patienten profitierten in Bezug auf Lungenfunktion, Belastung, Dyspnoe und Lebensqualität. Der beschriebene deutliche Effekt setze aber eine sorgfältige Auswahl der Patienten sowie ausreichende Erfahrung im Umgang mit möglichen Komplikationen der Intervention voraus, schreiben Kemp et al..

Dr. med. Susanne Meinrenken, Bremen 